\title{
Utilization of evidence theory in the detection of salient regions in successive $\mathrm{CT}$ images
}

\author{
THANOS ATHANASIADIS ${ }^{1}$, MANOLIS WALLACE ${ }^{2}$, KOSTAS KARPOUZIS $^{1}$ and STEFANOS KOLLIAS $^{1}$ \\ ${ }^{1}$ Image, Video and Multimedia Systems Laboratory, National Technical University of Athens, \\ 9 Heroon Polytechniou Street, GR-157 80 Athens; ${ }^{2}$ Department of Computer Science, \\ University of Indianapolis, Athens Campus, 9 Ipitou Street, GR-105 57 Athens, Greece
}

Received September 6, 2005; Accepted September 29, 2005

\begin{abstract}
This study presents an integrated approach to locating and presenting the medical practitioner with salient regions in a computed tomography (CT) scan when focusing on the area of the liver. A number of image processing tasks are performed in successive scans to extract areas with a different features than that of the greater part of the organ. In general, these areas do not always correspond to pathological patterns, but may be the result of noise in the scanned image or related to veins passing through the tissue. The result of the algorithm is the original image with a mask indicating these regions, so the attention of the medical practitioner is drawn to them for further examination. The algorithm also calculates a measure of confidence of the system, with respect to the extraction of the salient region, based on the fact that a region with a similar pattern is also located in successive scans. This essentially represents the hypothesis that the volume of both pathological patterns and blood vessels, but not noise patterns, is large enough to be captured in successive scans.
\end{abstract}

\section{Introduction}

The ever-improving capabilities of medical image acquisition and computer vision technology are fast introducing a number of interactive intervention applications for medical image analysis researchers and practitioners. In this framework, integrated approaches fuse intelligent techniques such as fuzzy logic or evidence theory with lower level image processing algorithms to provide tools that locate and indicate specific patterns in medical images to surgeons; this process must also incorporate additional requirements, i.e. information from other sources, such as preoperative images,

Correspondence to: Dr Thanos Athanasiadis, Image, Video and Multimedia Systems Laboratory, National Technical University of Athens, 9 Heroon Polytechniou Street, GR-157 80 Athens, Greece E-mail: thanos@image.ntua.gr

Key words: computed tomography images, salient regions, image segmentation, evidence theory, minimum uncertainty principle and operate as closely to real-time as possible. In addition, the registration of findings in different types of information poses additional concerns.

In this study, we present an integrated approach to locating salient regions in successive CT images and using the evidence theory to associate a confidence value to any abnormalities before alerting the practitioner. In a typical scenario, multiple successive CT scans from a specific patient are available and processed separately. Abnormalities are modeled as distinct areas with a different pattern, located in at least three consecutive scans; this heuristic is imposed to help eliminate erroneous detection by increasing the confidence of results with respect to the number of slices found in an alerting area and the percentage of overlap in the respective areas. According to the minimum uncertainty principle, the combination of evidence from independent sources, such as results from the processing of different slices, reduces overall uncertainty and augments overall confidence $(1,2)$.

The main contribution of our approach is that the minimum uncertainty principle and evidence theory are both utilized when processing raw medical images. Thus, despite starting with low resolution images and well-established medical image processing techniques, the final result is accompanied by remarkably high confidence due to combining the results of processing distinct slices acquired independently and analysing data using the Dempster-Shafer theory (2).

\section{Materials and methods}

Images. Images were acquired with a 16-multislice CT scanner at $120 \mathrm{kV}, 294 \mathrm{~mA}$ using a B31F kernel. The field of view was $36.9 \times 36.9 \mathrm{~cm}$ with a $512 \times 512$ matrix, and the slice thickness was $2.0 \mathrm{~mm}$, with a collination of $1.5 \mathrm{~mm}$.

Image segmentation. Our goal is to extract salient regions of the liver using a hybrid segmentation algorithm. Image segmentation algorithms are generally based on 1 of 2 basic properties of intensity values: discontinuity and similarity. In the first category, the approach is to partition an image based on abrupt changes in intensity, such as edges in an image. The principal approaches in the second category are based on partitioning an image into regions that are similar according 
to a set of predefined criteria. Thresholding, region growing and region splitting and merging are examples of methods in this category.

After enhancing the contrast of the image using morphological operators, we extract the extended minima of the image, i.e. regions of the image that are regional minima and whose external boundary pixels all have a value greater than the threshold. We also extract extended maxima following the dual procedure. Using the knowledge that luminance is proportional to tissue density, the aboveextracted regions are of great interest for further analysis. We utilize a watershed segmentation algorithm using the above regions as markers $(3,4)$. The main idea of the watershed algorithm is that the gradient of the image is created, then 'flooded' starting from the local minima (catchment basins) until reaching the watershed edges, which separate two different basins. The output, an over-segmented image, is further improved with a color recursive shortest spanning tree (RSST) (5) that roughly defines the borders of the liver. RSST belongs to the region growing segmentation techniques and merges neighboring segments according to a similarity criterion (which in our case is luminosity). Given the mask of the liver and salient regions previously detected, we allow a post-processing step to refine those regions. This refinement also uses well-established morphological processing techniques discussed later in this study.

This process is followed for three successive CT images. The salient regions extracted in each image are superimposed one over the other in order to check whether certain regions are found in this sequence and to what degree. If this is the case, then we can safely assume that this salient region is a salient volume. In any other case, we assign a much smaller degree of salience to the region or even completely dispose of it.

Morphological processing. In this study, we utilize morphological operators to enhance the quality of input images and refine the final results. Mathematical morphology is a theory concerned with the processing and analysis of images, using operator and functionals based on topological and geometrical concepts (6). Regarding the enhancement, tophat and bottom-hat operators are used in order to increase the contrast of the image. For the second case, reconstruction opening is used to eliminate unwanted, false-detected regions based on either their small size or position exactly at the perimeter of the liver (we suppose those regions to be false due to segmentation defects). Further, the closing operator is used to close possible holes in the salient regions in a gentle manner without smoothing their shape.

Evidence theory. We utilize the a priori knowledge that an abnormality in most cases is not plane, but has a smaller or bigger volume, which means that the destroyed tissue is tracked in more than one image. The surface of the abnormality differs in each subsequent image, and it is normal to expect small changes as the axial analysis is high enough. In other words, there is a good amount of useful information correlated between successive CT images. We expect that an abnormal region spreads in the image sequence, depending on the size of its volume.

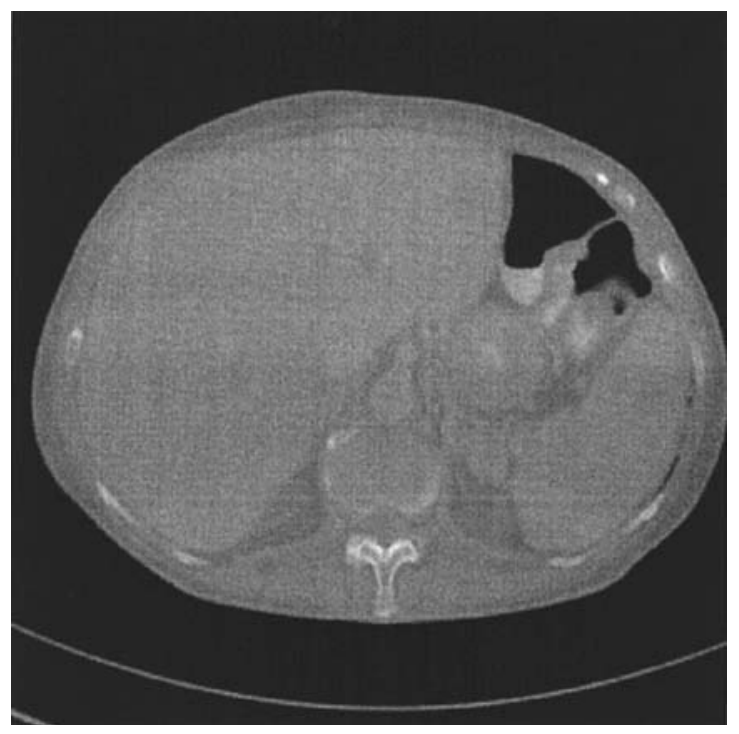

Figure 1. Input image.

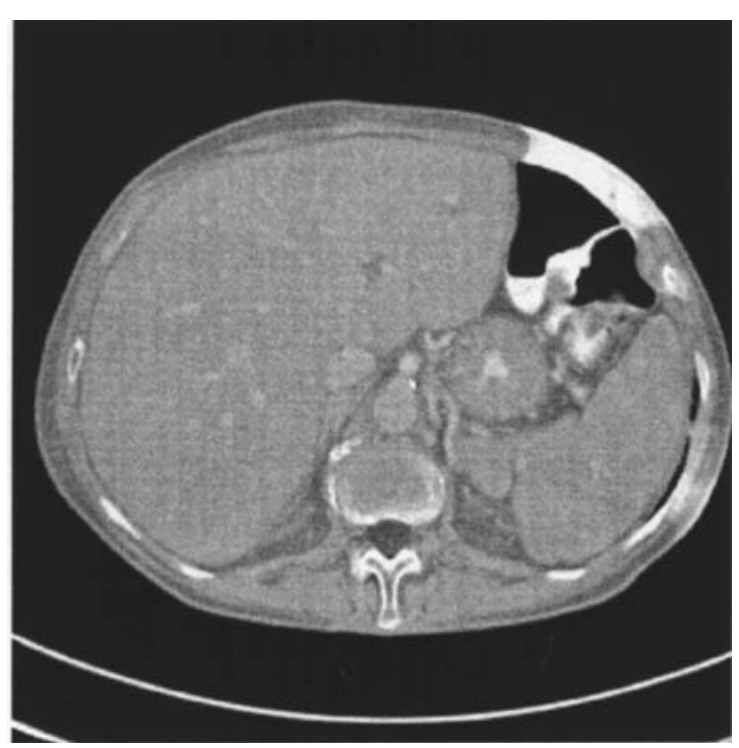

Figure 2. Image after contrast enhancement.

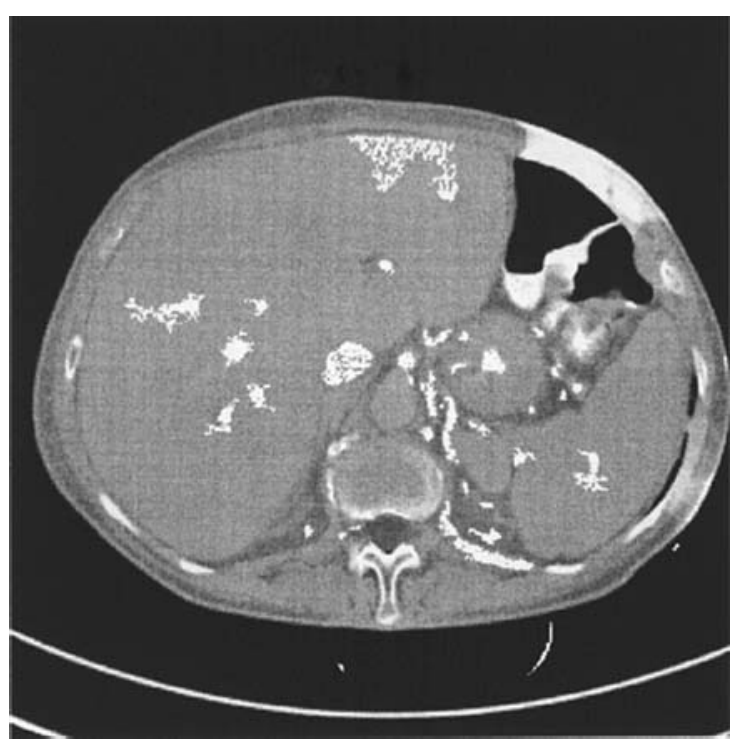

Figure 3. Extended markers superimposed on the input image. 


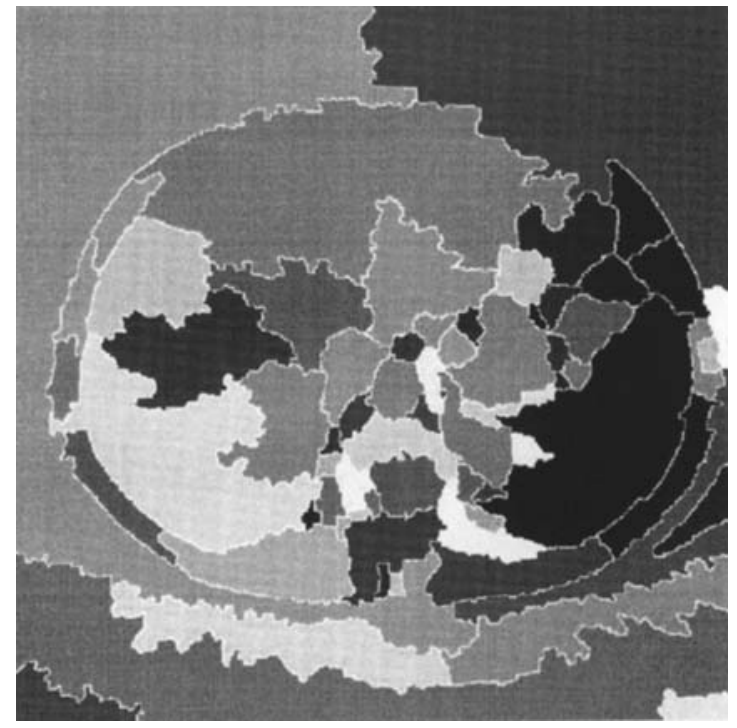

Figure 4. Result of watershed segmentation.

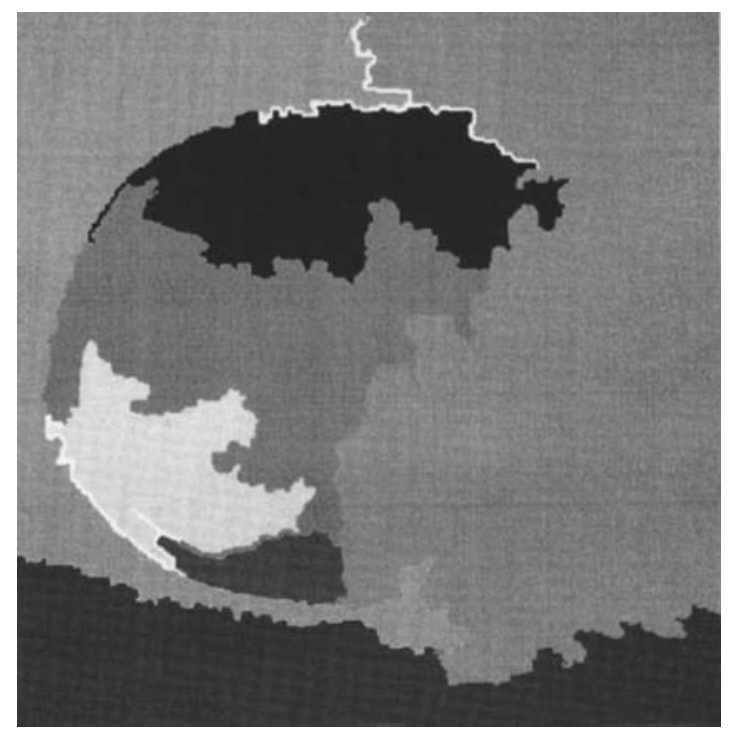

Figure 5. Result of the RSST algorithm.

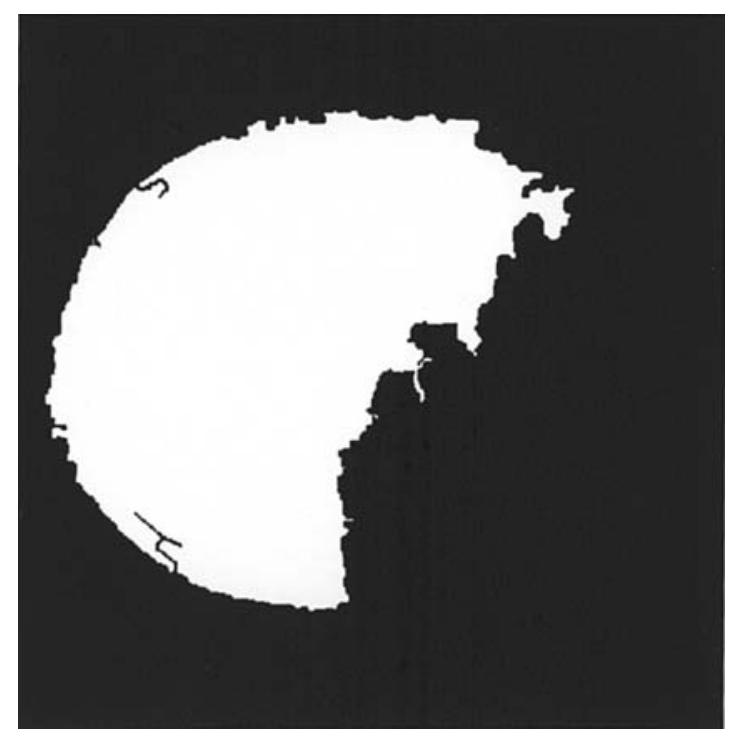

Figure 6. Binary mask segmentation of the liver.
Possibility theory, specifically its branch called evidence theory, provides the mathematical tools through which we can model and process this kind of information (1). In this modeling, we consider each frame as a carrier of evidence concerning the state of the examined organ. Thus, if the texture analysis of a frame identifies a suspicious region, then we consider the specific frame to offer evidence that there is a problem in the organ. The confidence in this evidence is estimated through the certainty and clarity of image processing results, as well as through the intensity of differences between the measured texture and that of the healthy organ model.

Having analyzed multiple frames of the same organ is similar to having questioned different people about the same event: the evidence provided by these independent sources needs to be combined in a way that also considers their initial certainty, with some sources of evidence providing higher certainty than others in order to reach an overall estimation. This is done using the Dempster-Shafer formula, which is the most established and widely used approach to combining evidence.

Specifically in our domain, the evidence from different frames can only be considered when it derives from consecutive frames and identifies the candidate region of interest in roughly the same position in each frame; otherwise, the two pieces of evidence are not referenced in the same volume and should not be combined. In this way, false positives due to texture variations occurring for different reasons, such as the detection of blood vessels, can be avoided.

For successive images, this is integrated in our automated analysis system as a rule that the surface of abnormal regions should be: 1) of similar size; and 2) at the same approximate coordinates $\{\mathrm{x}, \mathrm{y}\}$. This improves efficiency of the system by eliminating incorrect classifications of abnormal regions. Thus, the examination of successive images reduces the risk of a false positive due to incorrect extraction of parameters.

Minimum uncertainty principle. Despite the success of these approaches, several problems still inhibit large-scale automated use in robustly segmenting relevant structures in medical images over different datasets. For example, boundary localization algorithms are sensitive to variations in image acquisition parameters and initial starting positions. Since the domain in which these general-purpose algorithms are performed is known beforehand and well-documented, recognition systems would need to start incorporating the necessary knowledge into higher level reasoning notions and knowledge models. The minimum uncertainty principle provides the framework needed to overcome this.

According to the minimum uncertainty principle, the consideration of extra information not initially available cannot augment the uncertainty; it can only help alleviate it. Thus, the uncertainty contained in a process can be alleviated in part by considering more information. Porting this to our application domain, various well-known and established image processing algorithms are at times applied to a slice of the CT scans. The results are combined, while also considering the certainty for each individual result, which 


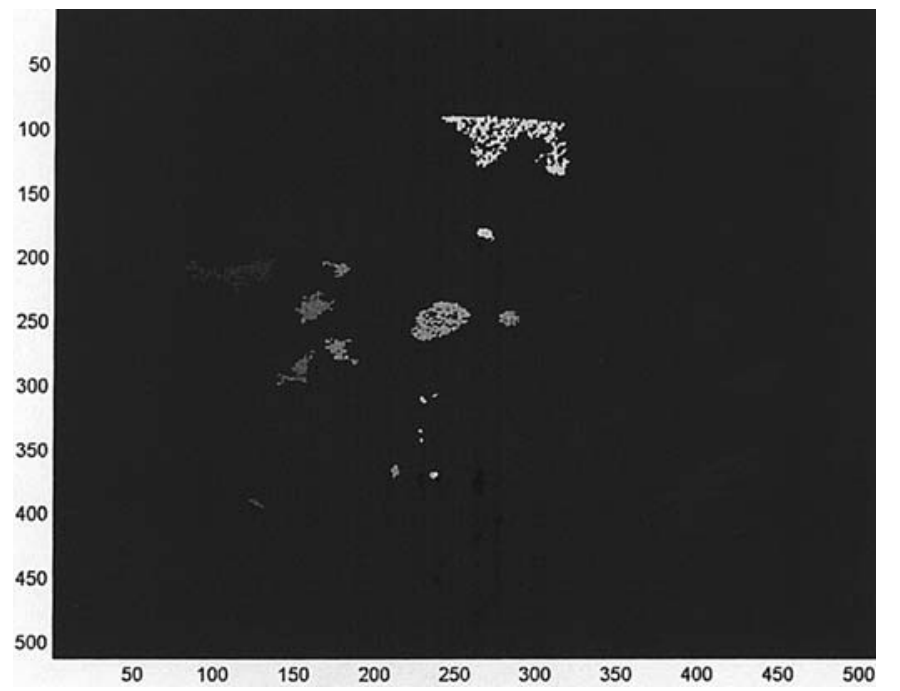

Figure 7. Possible salient regions inside the liver.

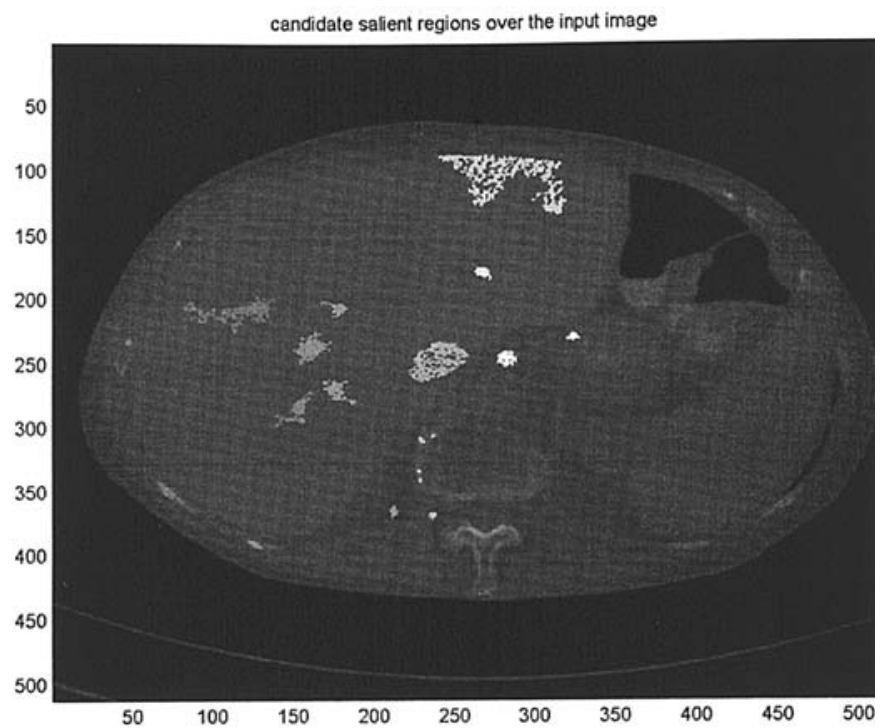

Figure 8 . Possible salient regions inside the liver superimposed over the input image.

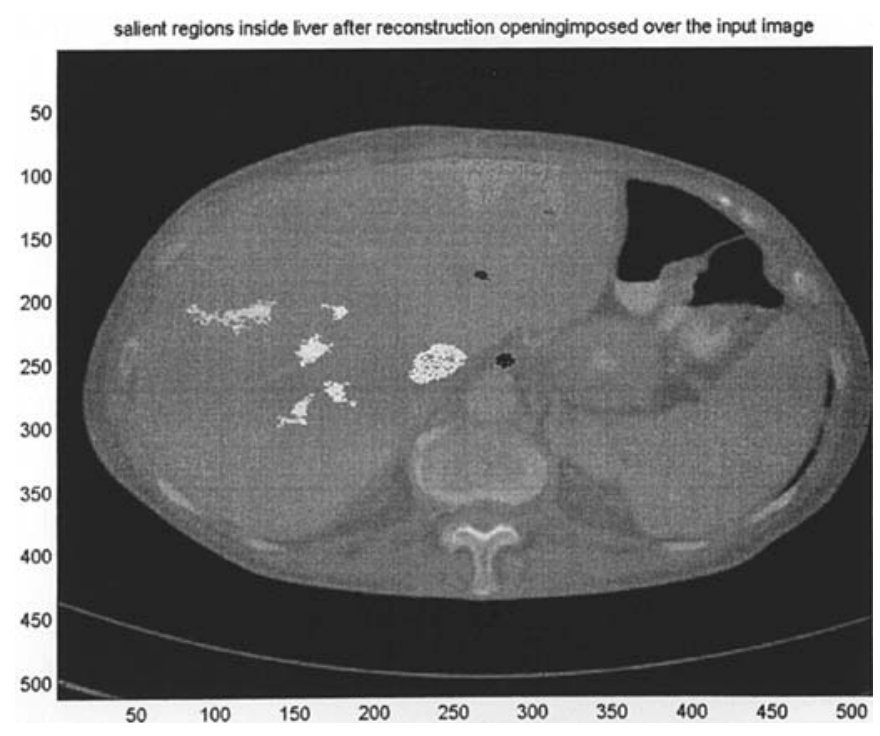

Figure 9. Salient regions after refinement. according to the minimum uncertainty principle assures us that in all cases the overall estimation is equivalent to, if not better than, the estimation provided by the best performing algorithm for a specific slice. More importantly, this algorithm might not always be the same for all slices.

\section{Results}

Among the test set of approximately 700 images, we selected three consecutive slices at the approximate height of the liver. Fig. 1 shows the first of the three slices, and Fig. 2 shows the same image just after contrast enhancement. Fig. 3 depicts the initial markers (colored white) for the whole image superimposed over the input image.

Figs. 4 and 5 show the results of two segmentation algorithms, watershed and RSST, respectively. It is evident that the over-segmented result of the watershed is practically useless, whereas the color-based RSST produces a more meaningful segmentation.

Since our focus is on the liver, we can utilize a priori knowledge of its position and size. Therefore, we can merge the segments produced from RSST that compose the liver and create its binary mask, as illustrated in Fig. 6.

Combining the mask of the liver with possible salient regions extracted previously, we isolate only those inside the boundaries of the liver. Fig. 7 indicates those regions, while the image mask of the liver area lies in the background. Fig. 8 shows the same regions superimposed over the input image.

Figs. 1-8 depict the results prior to post-processing refinement. Following the method explained above, we created Fig. 9, which shows the remaining salient regions after the reconstruction opening that eliminated false regions (those of minute size and those at the perimeter of the liver).

Fig. 10 demonstrates the final results of our study. The first three pictures (Fig. 10A-C) are the result of Fig. 9 after a soft closing that fills the holes of salient regions for the three consecutive slices. The last image (Fig. 10D) shows a combination of the three successive masks of candidate salient regions. Note that some of the candidate salient regions of the first three images have been eliminated since they failed to meet the criterion of matching respective areas in successive slices. For example, the large region in the top center of the two first images is not part of the final image since it disappears in the third image.

\section{Discussion}

Initial studies in the area of computer vision-based medical image processing were mainly focused in segmenting specific structures and labeling them as known objects, registering two different image datasets, and calculating parameters to describe cardiac motion from image sequences (7). A typical application in segmentation was to first detect contrast edges from two-dimensional image datasets and perform edge grouping or linking operations to come up with object boundaries $(8,9)$. In most cases, general purpose computer vision algorithms are transformed to comply with the specific requirements of medical images; as a result, issues related to both general and object-specific segmentation are still abundant in low-level processing concepts, typically tackled 

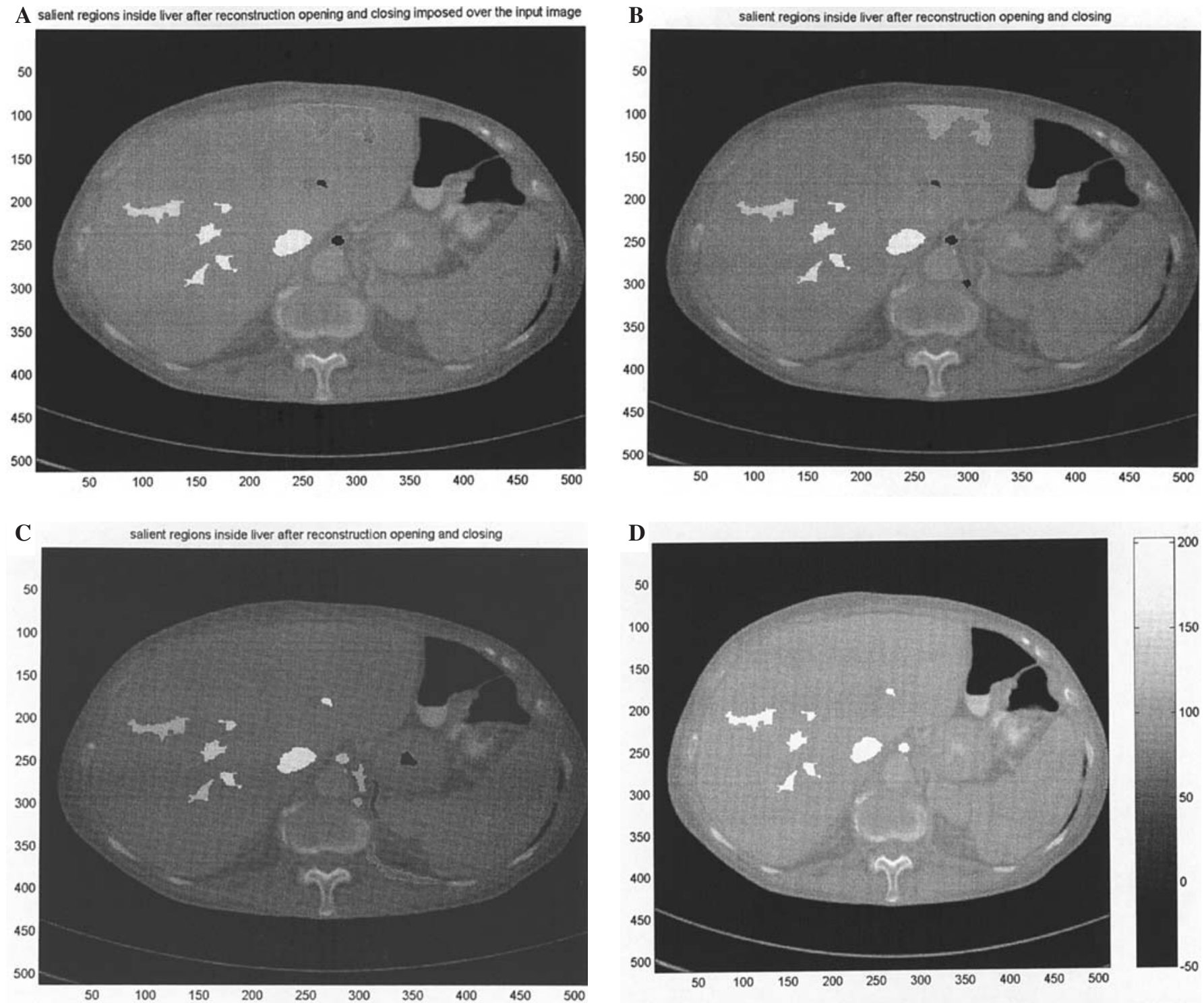

Figure 10. (A) Salient regions after morphological closing that filled holes in the 1st slice. (B) Salient regions after morphological closing that filled holes in the 2nd slice. (C) Salient regions after morphological closing that filled holes in the 3rd slice. (D) Combined salient regions after morphological closing that filled the holes.

with boundary estimation and region growing schemes. Seminal approaches in the former problem are based on deformable models (10), especially when transformed to tackle specific medical imaging problems (in 3D) (3) and gradient vectors controlling contour (in 2D) or surface deformation (11).

Although there is no strict definition regarding texture modeling, it is something commonly found in nature and in a microscopic view of human beings. Human tissues have a well-defined structure that differentiates one from another. Various abnormalities, such as cancer cells, hematomas and cysts, are visually separable, since these tissues have different structures, and thus texture $(12,13)$. In general, image texture is proposed as a useful grouping and quantization measure, especially in ultrasound or CT image data (14) or echocardiograms (15). In our approach, we assume that most human organs in a CT image are homogeneous in texture, since each organ has a standard structure of cells and tissue density. The above assumption, in addition to the fact that luminance is proportional to the tissue density in CT images (i.e. bone tissue, having more density, appears brighter than muscle tissue), facilitates the task of segmentation. Therefore, the boundaries of each organ are quite distinct.

In our approach, we extend the above-mentioned studies by utilizing the minimum uncertainty principle and evidence theory in order to tackle the processing of low resolution images. This extension is necessary since the lack of detailed information in the CT images makes it difficult to determine texture and matching algorithms to extract precise, meaningful information with a high degree of confidence. Introduction of the evidence theory in essence enables our approach to combine findings in successive slices, with the assumption that the volume of a salient region spans more than two of these slices. This assumption enhances the systematic image processing steps and provides the final result with a confidence value; as a result, the system response can be validated both internally and by the medical practitioners. 


\section{References}

1. Klir G and Yuan B: Fuzzy sets and fuzzy logic, theory and applications. Prentice Hall, New Jersey, 1995.

2. Shafer G: A Mathematical Theory of Evidence. Princeton University Press, Princeton, NJ, 1976.

3. Beucher S and Meyer F: The morphological approach to segmentation: the watershed transformation. In: Mathematical Morphology in Image Processing. Doughertty ER (ed). Marcel Dekker, New York, NY, 1993.

4. Meyer F and Beucher S: Morphological segmentation. J Visual Commun Image Rep 1: 21-45, 1990.

5. Kwok SH and Constantinides AG: A fast recursive shortest spanning tree for image segmentation and edge detection. IEEE Trans Image Process 6: 328-332, 1997.

6. Heijmans H: Morphological Image Operators. Academic Press, San Diego, CA.

7. Duncan J and Ayache N: Medical image analysis: progress over two decades and the challenges ahead. IEEE Trans Pattern Anal Machine Intelligence 22: 85-106, 2000.

8. Maglogiannis I: Automated segmentation and registration of dermatological images. J Math Model Algorithms 2: 277-294, 2003.
9. Yachida M, Ykeda M and Tsuji S: A plan-guided analysis of cineangiograms for measurement of dynamic behavior of the heart wall. IEEE Trans Pattern Anal Machine Intelligence 2: $537-543,1980$.

10. McInerney T and Terzopoulos D: Deformable models in medical image analysis: a survey. Med Image Anal 1: 91-108, 1996.

11. Xu C and Prince J: A Generalized gradient vector flow for active contour models. In: Proceedings of the Conference on Information Sciences and Systems. pp885-890, 1997.

12. Mir AH, Hanmandlu M and Tandon SN: Texture analysis of CT-images for early detection of liver malignancy. Biomed Sci Instrum 31: 213-217, 1995.

13. Tourassi G: Journey toward computer-aided diagnosis: role of image texture analysis. Radiology 213: 317-320, 1999.

14. Meunier J and Bertrand M: Ultrasonic texture motion analysis: theory and simulation. IEEE Trans Med Imaging 14: 293-300, 1995.

15. Morris D: An evaluation of the use of texture measures for tissue characterization of ultrasound images of in vivo human placenta. Ultra Med Biol 14: 387-395, 1988. 